Revue des patrimoines

\title{
Des chevaux sous l'Occupation : le charnier du site archéologique des Varennes à Bar-sur-Aube (Aube)
}

Gilles Deborde

\section{OpenEdition}

Journals

Édition électronique

URL : http://journals.openedition.org/insitu/12150

DOI : 10.4000/insitu. 12150

ISSN : 1630-7305

Éditeur

Ministère de la culture

Référence électronique

Gilles Deborde, «Des chevaux sous l'Occupation : le charnier du site archéologique des Varennes à Bar-sur-Aube (Aube) », In Situ [En ligne], 27 | 2015, mis en ligne le 27 octobre 2015, consulté le 03 mai 2019. URL : http://journals.openedition.org/insitu/12150 ; DOI : 10.4000/insitu.12150

Ce document a été généré automatiquement le 3 mai 2019.

\section{(c) (i) () $\Theta$}

In Situ Revues des patrimoines est mis à disposition selon les termes de la licence Creative Commons Attribution - Pas d'Utilisation Commerciale - Pas de Modification 4.0 International. 


\title{
Des chevaux sous l'Occupation : le charnier du site archéologique des Varennes à Bar-sur-Aube (Aube)
}

\author{
Gilles Deborde
}

\section{Présentation}

1 Pour l'historien attaché à l'étude des faits de société, l'archéologie peut être perçue comme une discipline plutôt portée à révéler des faits divers, trop souvent funestes, ou simplement destinée à illustrer les livres d'histoire. Si, dans la société actuelle soumise au pouvoir pernicieux de l'image, la tendance à forcer le second trait est patente, la pertinence d'une exploration systématique de faits isolés, encore imprimés dans les sols et les paysages, est revendiquée par une archéologie moderne. L'exemple de la fouille du site des Varennes à Bar-sur-Aube illustre l'intérêt d'une étude pluridisciplinaire de ce qui aurait pu apparaitre de prime abord comme le produit d'un fait divers contemporain, mais qui a ouvert le champ à une enquête historique sur un aspect méconnu du rôle des troupes d'occupation dans l'Aube à la fin de la Seconde Guerre mondiale.

\section{Le contexte archéologique}

2 L'Institut national de recherches archéologiques préventives (INRAP) a conduit en 2013, avant la construction d'un lotissement, la fouille d'une surface de $8000 \mathrm{~m} 2$ à la sortie de la ville de Bar-sur-Aube (fig. 1). L'intervention était justifiée par la mise au jour préalable et l'étude d'un tronçon de voie antique. 


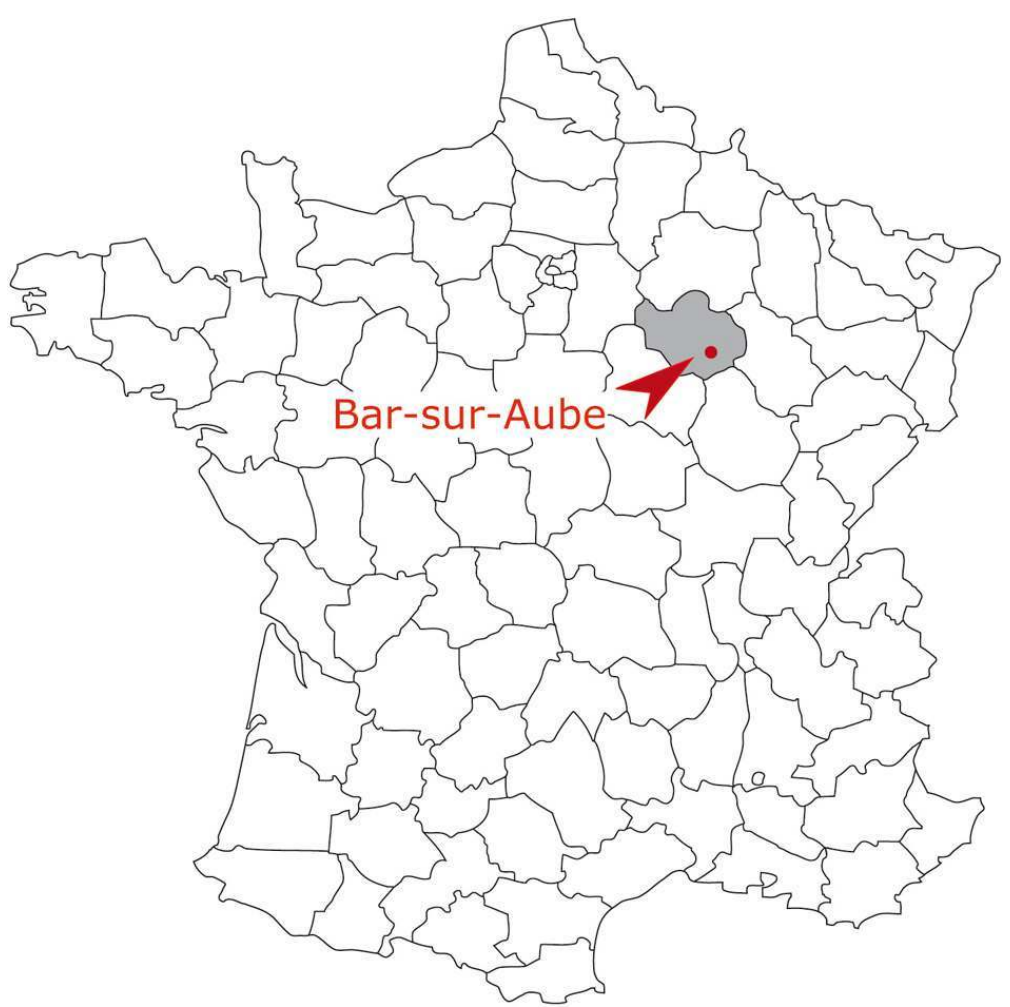

Carte de localisation de Bar-sur-Aube.

\section{La tranchée}

3 À l'une des extrémités du site est apparu en plan, creusé perpendiculairement à la voie romaine, le tracé crénelé d'une tranchée développée au-delà de la largeur de l'emprise étudiée, soit sur plus de $46 \mathrm{~m}$. Un sondage mécanique, destiné à étudier la structure de la voie, a atteint un diverticule de la tranchée, permettant ainsi de remarquer la présence, à l'intérieur, d'ossements de chevaux (fig. 2). 
Figure 2

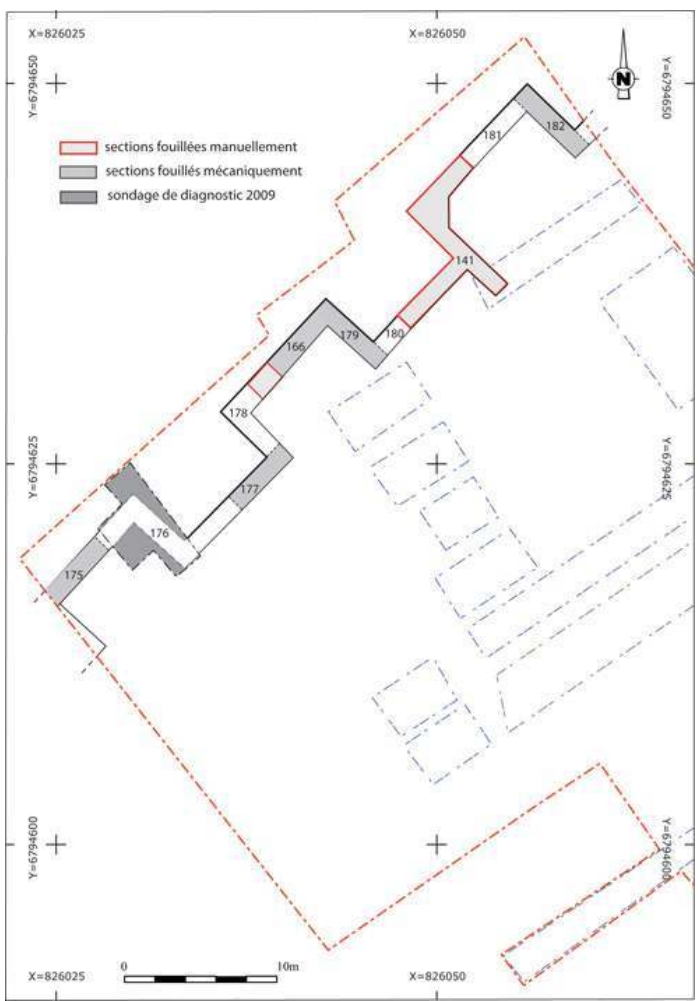

Position et extension de la tranchée 140 sur l'emprise de la zone fouillée. Infographie Deborde, Gilles. (C) Inrap, 2014.

4 Il est rapidement apparu que la tranchée, large de 1,20 m, pouvait ne contenir que des carcasses d'équidés. La fouille manuelle d'un volume de $20 \mathrm{~m} 3$ sur un premier tronçon (section 141) a permis d'identifier la présence de 17 individus complets disposés partiellement les uns sur les autres sur la hauteur de l'excavation.

Dans le temps imparti à l'opération, les autres sections ont dû être sondées mécaniquement et n'ont pu être prélevés que les os longs et les crânes, ceci à des fins biométriques et statistiques, corpus représentant 13 autres individus. S'il n'a pas été possible de vérifier sur ces autres sections une même simultanéité de fait, celle-ci reste toutefois probable, la position des individus dans la tranchée suivant une même organisation (fig. 3, fig. 4). 
Figure 3

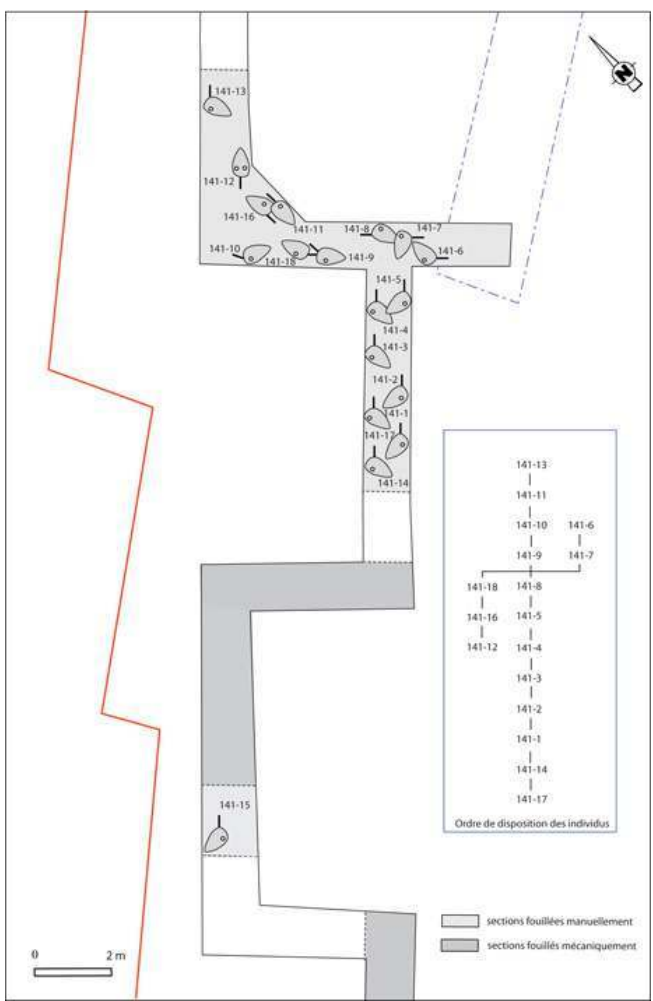

Position des chevaux à l'intérieur de la section de tranchée 141.

Infographie Deborde, Gilles. (C) Inrap, 2014. 


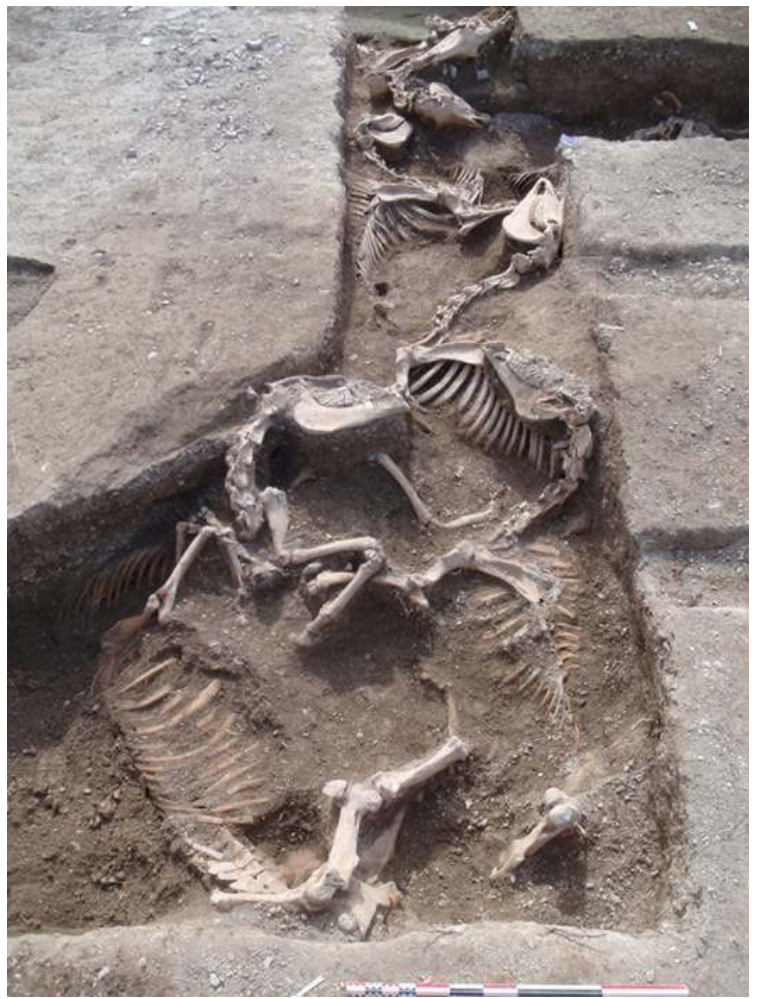

Disposition des carcasses à l'intérieur de la section de tranchée 141.

Phot. Deborde, Gilles. (c) Inrap, 2014.

On peut ainsi évaluer à 42 le nombre minimum d'équidés inhumés de manière opportune dans cette fosse dont on ignore la longueur exacte.

En fin d'intervention, nous avons pu établir une relation entre cette tranchée et une fosse mise au jour plus au sud à l'occasion d'un diagnostic archéologique préalable en 2009. Il s'agissait en fait d'une section de la tranchée remblayée avec des déchets métalliques et culinaires (section 176). Nous disposions dès lors d'une chronologie relative permettant de réduire considérablement le champ culturel et historique de l'étude.

\section{Les artefacts}

Dans la section 141, plusieurs chevaux conservaient des fers aux pieds. Les plus grands fers sont caractérisés par la présence de mortaises disposées aux extrémités des éponges et sur la pince. Deux d'entre eux se distinguent par l'ajout sur leur face externe d'une plaque en métal ajustée à la largeur et à la longueur du fer, et relevée à angle droit à l'arrière du pied. Cette plaque était maintenue sur les éponges par deux crampons et latéralement par une languette rabattue sur la face interne de la pince (fig. 5). 
Figure 5

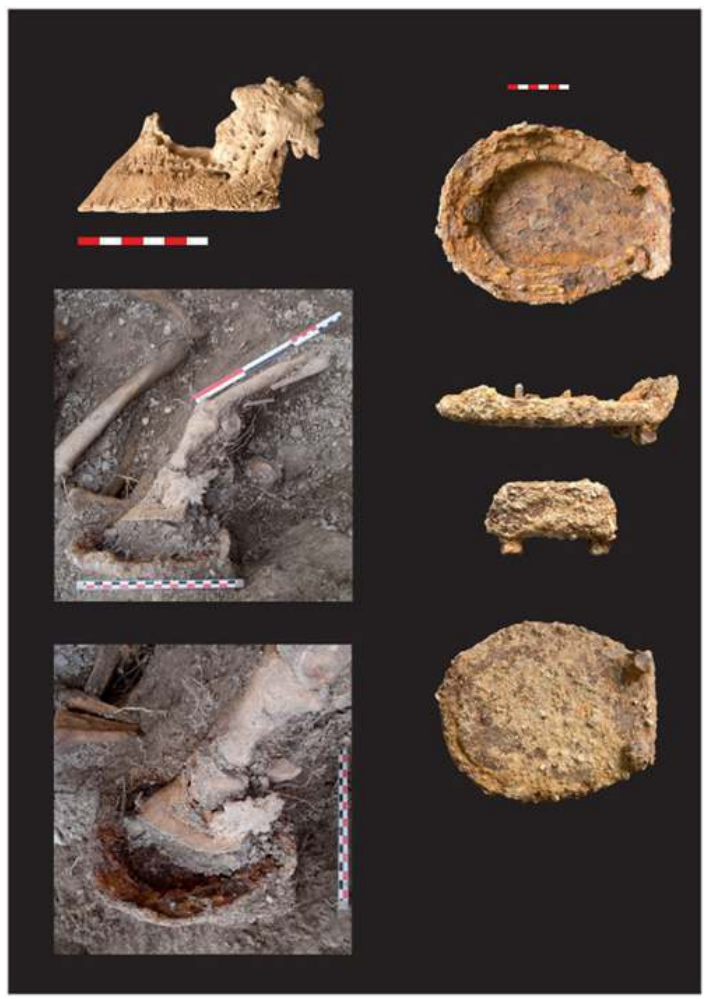

Fer à plaque et ossification des cartilages ungulaires de l'individu $n^{\circ} 4$.

Phot. Bandelli, Alessio. (c) Inrap, 2014

Parmi les objets contemporains du dépôt de chevaux de la section 176 figure une semelle de brodequin en caoutchouc estampillée MORVAN, une tasse en faïence de Sarreguemines et une cartouche de fusil. La société Morvan, à Château-Chinon, a produit pour l'armée française en 1938 des masques à gaz en caoutchouc puis, à partir de 1941 pour l'armée allemande, des semelles et des talons, également en caoutchouc et ainsi estampillés ${ }^{1}$. La tasse en faïence blanche de Sarreguemines fait partie des productions à décors séditieux de l'atelier d'Alexandre Geiger durant l'annexion allemande entre 1871 et $1918^{2}$. La cartouche est une munition française de $8 \mathrm{~mm}$ marquée au culot et fabriquée en 1929 par la Société française des munitions (Gevelot) à Paris. Ce type de munitions était utilisé exclusivement par l'armée et se retrouvait dans les fusils de type Lebel, les mousquetons Berthier et les mitrailleuses Hotchkiss. Hormis la résistance et la gendarmerie, qui conserva les mousquetons Berthier jusqu'à la fin des années 1960, ce type de munitions reste marginal dans l'armée française de libération en 1944, alors équipée de matériel anglais ou américain ${ }^{3}$.

\section{Le contexte historique}

10 Avec ce matériel contemporain associé, le plan de la tranchée pouvait désormais présenter les caractères d'un ouvrage de défense passive tel que préconisé par l'administration française à partir de 1940 pour assurer la protection de la population civile. Ce type d'ouvrage enterré fut également mis en œuvre par les Allemands sur des sites civils et militaires pour protéger les troupes d'occupation. 


\section{L'usine des Forges}

11 Si la tranchée des Varennes présente toutes les caractéristiques d'une tranchée de défense passive de la Seconde Guerre mondiale, à qui était-elle destinée ?

12 De l'autre côté de la route nationale, face à cette tranchée, se trouvent encore les installations de l'entreprise Manoir Industrie, ancienne usine des Forges spécialisée dans le façonnage de pièces métalliques. Cette usine, créée en 1902, ne connut aucune interruption d'activité durant les deux conflits mondiaux et s'est largement développée après 1950. D'anciens ouvriers baralbins devaient certainement avoir encore en mémoire la présence de ces tranchées destinées à les protéger des bombes et des gaz.

$13 \mathrm{Ni}$ les articles parus dans la presse locale, ni les images commentées sur le site Internet de l'Inrap 4 à l'issue de la fouille ni les interviews réalisées auprès d'anciens ouvriers de l'usine n'ont permis d'obtenir la moindre information sur l'origine de cette tranchée et sur le sort de ces chevaux.

\section{La ferme du Chalet}

14 Sur la section E du cadastre communal de $1837^{5}$, l'emprise fouillée peut être précisément localisée en bordure de la Route royale $\mathrm{n}^{\circ} 19$ de Paris à Bâle, dite également route d'Ailleville. Ce document parcellaire, antérieur à la fondation de l'usine, a servi de base de calcul de l'impôt jusqu'au milieu du $\mathrm{xx}^{\mathrm{e}}$ siècle et pouvait en conséquence nous permettre de retracer l'historique des possessions foncières sur le secteur (fig. 6). 


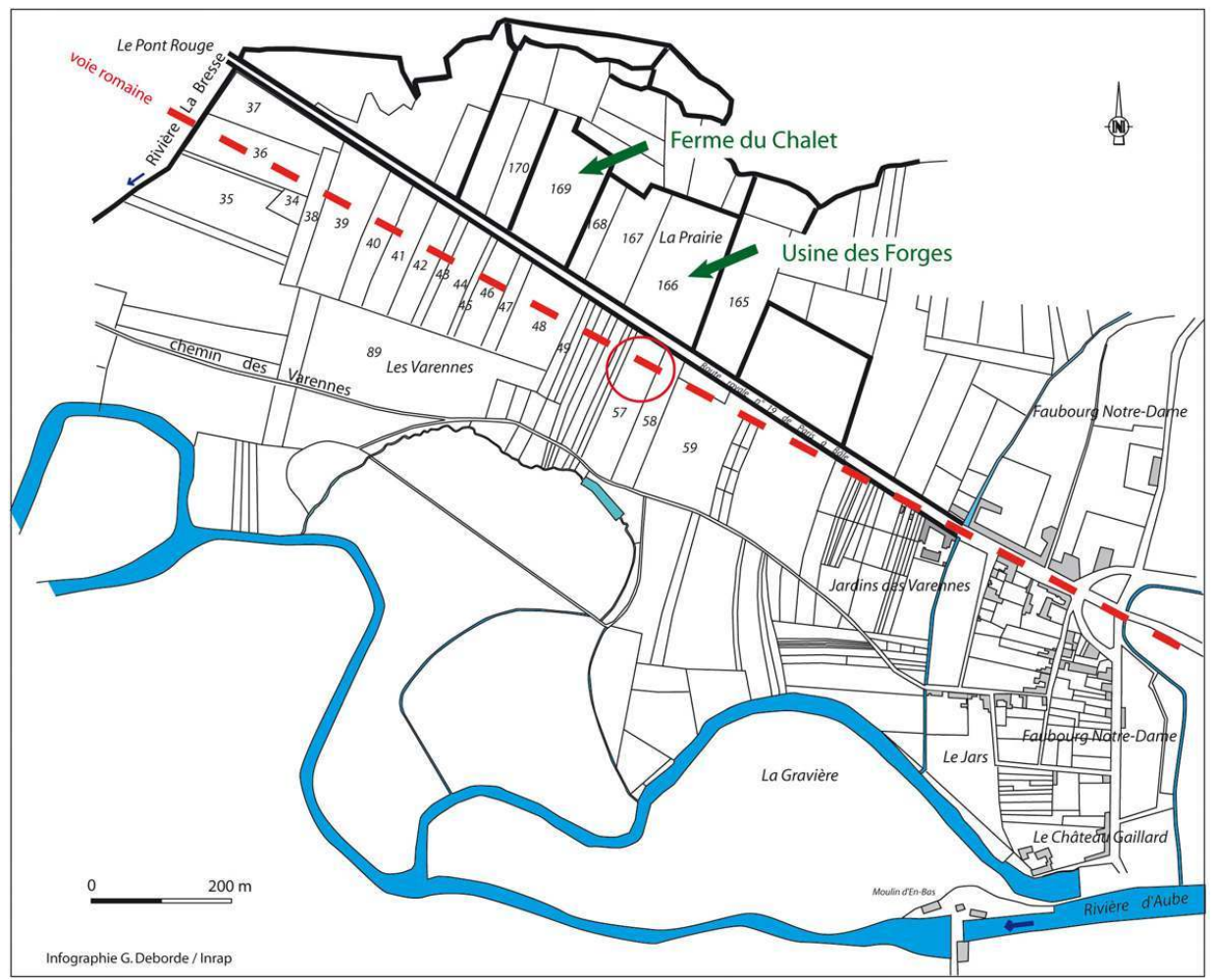

Localisation de l'usine des Forges et de la ferme du Chalet au nord du site des Varennes sur fond du cadastre de 1837.

Infographie Deborde, Gilles. (C) Inrap, 2014.

C'est dans les anciennes matrices foncières de la commune de Bar-sur-Aube versées aux archives départementales de l'Aube que nous avons trouvé un premier indice déterminant. La matrice des propriétés bâties relatives à la section $\mathrm{E}$, parcelle $169^{6}$, fait état de la présence d'une maison, enregistrée en 1911 comme appartenant à Mme veuve Tripotin et ses fils Jules et Just. En 1940, cette propriété bâtie est enregistrée au seul nom de Just Tripotin, à l'adresse de la «ferme du Chalet » située route d'Ailleville à Bar-surAube. En 1943, la propriété de la maison attachée à la parcelle 169 passe dans le patrimoine des Forges de Bar-sur-Aube, l'usine d'estampage occupant alors depuis 1932 les parcelles voisines E 166 et 167. En 1945, Just Tripotin a cédé sa propriété et est domicilié chez un habitant de Bar-sur-Aube, faubourg de Paris.

Parmi les archives non encore classées de la mairie de Bar-sur-Aube, nous avons pu retrouver d'autres matrices cadastrales plus anciennes que celles déposées aux archives départementales. Elles nous ont appris que la ferme du Chalet, officiellement localisée au lieudit la Prairie, était entrée au registre des propriétés bâties en 1882, ce qui justifie son absence sur le cadastre de 1837.

Nous avons consulté par ailleurs de nombreuses déclarations relatives à des "actes de piraterie » perpétrés par la Résistance sur la commune, à des dommages matériels causés par des bombardements sur la ville, à des pillages imputés aux troupes d'occupation en août 1944, à des demandes pour réparation de dommages de guerre et aux décisions d'engagement des indemnisations qui suivirent. 

la présence de l'usine d'estampage, elle n'a pu être comblée qu'à l'issue du conflit. Pourquoi un tel ouvrage n'aurait-il laissé aucun souvenir? Comment l'existence aussi récente de la ferme du Chalet peut-elle être ignorée, non seulement des habitants de Barsur-Aube mais également des inventaires historiques contemporains les plus exhaustifs? Comment la mise en terre d'un tel cheptel a-t-elle pu rester confidentielle?

\section{L'exploration de nouvelles pistes}

écemment mis à la disposition du public, les documents attachés au Bureau de liaison, échanges de courriers et de notes entre la sous-préfecture de Bar-sur-Aube, la préfecture et les autorités allemandes pour la période 1940-1944, constituent un fonds très riche offrant de multiples angles d'approche de l'histoire de l'Occupation. Une liasse concerne les réquisitions et les prêts de chevaux ${ }^{7}$, une autre intéresse les entreprises industrielles encore en activité pendant le conflit ${ }^{8}$.

\section{Le cheptel équin dans l'Aube sous l'Occupation}

On recense en 1939 dans le département de l'Aube un peu plus de 26000 chevaux aptes au travail. À la suite de la déclaration de guerre, les premières réquisitions au profit de l'armée française retirent à la culture ses meilleurs chevaux. La débâcle de 1940 va ensuite augmenter dans d'importantes proportions les pertes d'animaux de trait conduits sur les routes par leurs propriétaires. Après l'armistice du 22 juin 1940, le manque de carburant amène les autorités d'occupation à réquisitionner davantage de chevaux.

\section{Les réquisitions de chevaux entre 1940 et 1944}

21 À partir de juin 1940, dans l'Aube, plusieurs campagnes de réquisition sont effectuées chaque année. Les premières réquisitions consistent en un simple système d'échange: l'armée allemande prélève un animal valide et remet en échange à l'ancien propriétaire un cheval redescendu du front ou repris à l'armée française. Les chevaux malades ou blessés seront ainsi pour la plupart remis sur pieds par leurs nouveaux propriétaires avant d'être de nouveau échangés.

4000 chevaux sont touchés par ces transactions entre juin et décembre 1940. Les animaux prélevés sont regroupés et dirigés sur la gare de Troyes où ils sont embarqués. Il semble que le système fonctionne et ne perturbe pas trop les capacités de production agricole puisque, début 1941, environ 22500 chevaux sont encore employés sur les exploitations dans le département.

En avril 1941, l'autorité militaire allemande s'est dotée d'un service de soins aux chevaux qui va lui permettre de procéder, non plus à l'échange, mais à l'achat puis au prêt ou à la vente aux agriculteurs de bêtes valides. L'un de ces premiers hôpitaux vétérinaires ( Pferdlazareth) est installé à Bar-sur-Aube. À la fin du mois de mars 1941, le personnel de cet établissement, cantonné dans les bâtiments du collège, quitte le centre-ville pour une destination inconnue'. Les chevaux sont alors prêtés le plus souvent contre des avantages en nature: une douzaine d'œufs ou un poulet par semaine et par cheval. Quatre 
réquisitions par an en moyenne permettent de réapprovisionner la Wehrmacht en chevaux sains.

\section{La présentation des animaux}

Avant chaque réquisition, un recensement des chevaux disponibles dans chaque commune est effectué. Ne sont retenus que les chevaux de selle ou de trait «vigoureux, âgés de 4 à 10 ans, parfaitement sains, aptes à traîner et de bonne constitution ». Sont exclus de la présentation « les étalons, les juments pleines, les chevaux de trot et les purs sangs, les chevaux aveugles ou borgnes, les chevaux présentant des lésions à la jambe ou au sabot, ou atteints d'asthme ». Les équidés répondant à ces critères sont classés selon deux catégories : les chevaux de trait d'une hauteur à l'encolure ne dépassant pas 1,65 m, bien doués pour le pas et le trot, et les chevaux de selle d'une hauteur à l'encolure de $1,54 \mathrm{~m}$ à $1,60 \mathrm{~m}$, pouvant porter jusqu'à 100 kilos. Il n'était pas nécessaire que le cheval soit ferré, mais il devait être doté d'un licol et d'une longe.

Sur un cheptel donné susceptible de satisfaire les exigences de l'autorité allemande, un quart à un tiers environ est généralement prélevé à chaque réquisition. En mars 1942, 1500 chevaux sont présentés à l'occupant dans le département de l'Aube. Si l'on considère un recensement de 20613 chevaux de plus de trois ans encore présents dans les exploitations et les entreprises auboises en février 1943, seulement $7,5 \% \mathrm{du}$ cheptel étaient ainsi susceptibles d'être déplacés par leurs propriétaires jusqu'aux centres de révision.

En décembre 1943, ces regroupements sont mis en place dans sept centres et pour la première fois à Bar-sur-Aube, où 150 chevaux sont présentés sur la place de la Mairie pour un achat envisagé de 45 têtes.

La dernière réquisition de chevaux a lieu dans le département entre le 14 et le 17 août 1944. À Bar-sur-Aube, 25 chevaux devaient être achetés pour être embarqués ensuite en gare de Vendeuvre. Lors de cette ultime réquisition, sur 200 chevaux attendus à Troyes pour être présentés, seulement 15 furent amenés et achetés. Ce n'est donc pas le cheptel potentiellement acheté à Bar-sur-Aube cette semaine-là qui fut précipité dans la tranchée des Varennes.

Au travers de ce tableau (fig. 7) transparaît un fait que nous avons relevé avec intérêt. Tandis que les animaux sont logiquement regroupés sur des lieux d'embarquement parmi lesquels n'est jamais mentionnée la gare baralbine, les chevaux pris à Vendeuvre, le plus important cheptel du secteur, devaient être conduits jusqu'à Bar-sur-Aube pour y être présentés avant d'être, théoriquement, de nouveau reconduits à la gare de Vendeuvre pour être dirigés sur Troyes. Il devait y avoir, dans ce déplacement, une raison particulière que la documentation consultée ne précise pas mais qui n'est peut-être pas sans rapport avec la présence des chevaux dans la tranchée des Varennes. 


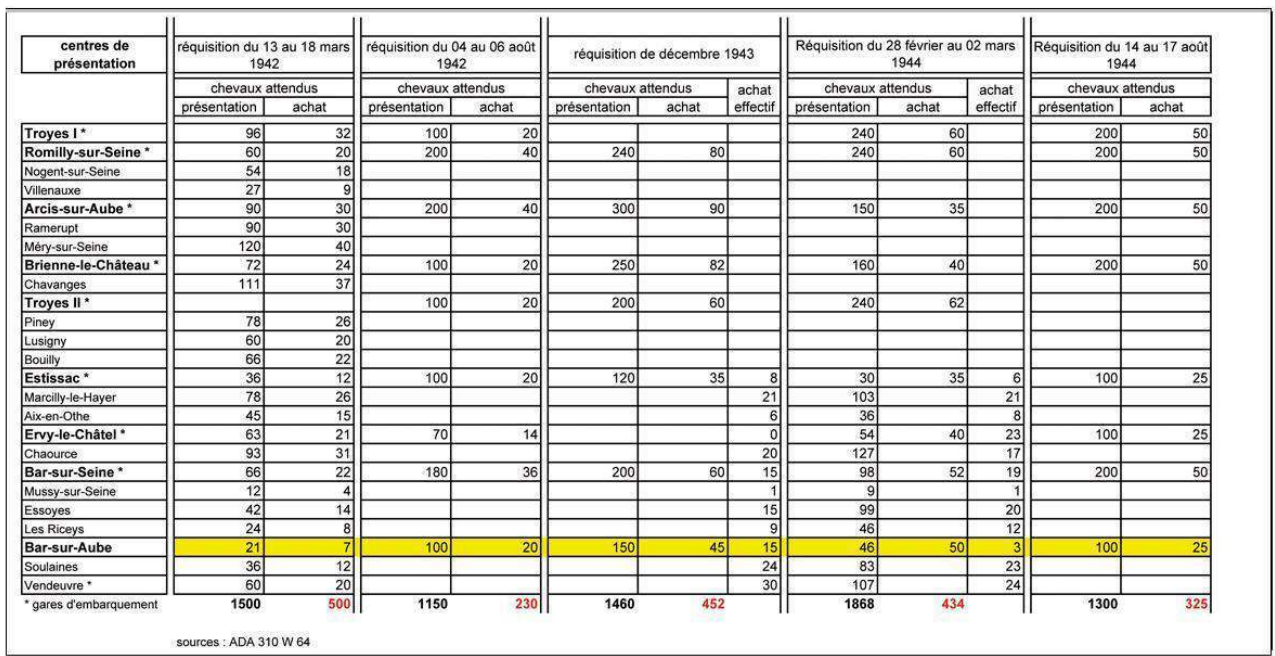

Quelques exemples d'effectifs de chevaux réquisitionnés et achetés durant l'Occupation dans les différents cantons du département de l'Aube.

Conception Deborde, Gilles. (c) Inrap, 2014.

\section{L'état sanitaire du cheptel}

Les recensements de chevaux présents sur les exploitations sont effectués à partir de fiches de signalement qui fournissent de précieuses informations sur le cheptel détenu par les agriculteurs et sur son état sanitaire. Une exploitation ne possède alors pas plus de quatre ou cinq chevaux, généralement d'âge avancé ( 15 ans en moyenne). Même si leur état sanitaire peut être déprécié pour dissuader les autorités allemandes d'un prélèvement préjudiciable pour l'exploitant, les chevaux sont souvent décrits comme galeux, pouilleux, boiteux, souffrant de maladies de peau ou de rhumatismes, portant de nombreuses cicatrices à l'encolure ou aux membres, causées par des éclats de bombes. D'autres sont borgnes ou aveugles, certains présentent des traumatismes nerveux (tics). D'une manière générale, ces animaux sont décrits comme "usés", " fatigués », " poussifs » ou « lymphatiques ", même si quelquefois, une jument de 25 ans en bonne santé peut faire exception ${ }^{10}$.

\section{La ville de Bar-sur-Aube sous l'occupation allemande}

Photographies et récits illustrent généreusement la période de la libération de la ville par les troupes alliées en août 1944. Les derniers chars allemands quittent le centre-ville et la population se réapproprie l'espace urbain. On peut légitimement comprendre que peu de documents relatent la période d'occupation, et c'est encore grâce à des archives, classées ou non, de la commune que nous avons pu recueillir quelques autres informations inédites sur la vie à Bar-sur-Aube durant ces années difficiles. 


\section{La défense passive et l'usine des Forges}

31 La poursuite de l'activité de l'usine au cours de la guerre était essentielle à l'économie militaire. L'usine des Forges fit donc partie des rares entreprises industrielles auboises placées, après juin 1940, directement sous la protection des autorités allemandes. Cette protection passait, bien entendu, par celle de la main-d'œuvre employée à la production.

Un premier rapport d'août-septembre 1942 établit une liste des entreprises industrielles auboises auxquelles sont imposées des mesures spéciales de défense passive. À cette époque, l'usine des Forges de Bar-sur-Aube jouit toujours d'un statut spécial qui ne permet pas de rendre publiques les informations la concernant. En juin 1943 toutefois, une première visite effectuée au sein de l'établissement est rapportée en préfecture. Ce rapport transmet les informations suivantes :

La totalité du personnel ( 55 personnes) serait dépourvue de masques à gaz. L'établissement devra se procurer les masques nécessaires pour le personnel de direction et les agents des équipes de défense passive. La protection collective du personnel, aussi bien contre les gaz que contre les effets des bombes explosives est à assurer dans une tranchée-abri à construire à l'intérieur de l'usine en un endroit judicieusement choisi, ayant une longueur utile de $12 \mathrm{~m}$ environ, dont les issues seraient fermées par des portes étanches, et qui serait munie d'un dispositif de ventilation avec prise d'air élevée à une dizaine de mètres de hauteur au moins.

Ce document fixe un ratio de $12 \mathrm{~m}$ de tranchée pour 55 personnes, proportion largement dépassée dans le cas de la tranchée des Varennes qui mesure, rappelons-le, plus de $46 \mathrm{~m}$ de longueur. Ces aménagements, s'ils ont été réalisés, n'ont laissé aucun souvenir aux ouvriers présents ou retournés à l'usine au lendemain de la guerre. Les tranchées de défense passive des Varennes auraient-elles donc eu une autre fonction?

\section{Les bombardements et autres dommages de guerre}

Sont conservés en mairie les déclarations concernant des actes de sabotage de réserves de foin et de fourrage et le bilan de quelques attaques aériennes. Quelques bombes sont tombées sur la gare en mai 1940 et juin 1944, mais le bombardement le plus important a eu lieu le 17 août 1944. Ce jour-là, quatorze bombes tombent sur la gare et deux bombes occasionnent des dégâts importants à l'usine des Forges ainsi qu'à la ferme du Chalet.

Les archives de la mairie de Bar-sur-Aube conservent également la totalité des mains courantes des décisions prises par la commission consultative intercommunale de dommages de guerre de Bar-sur-Aube créée le 21 mars 1945.

L'un de ces documents concerne l'usine des Forges, indemnisée le 12 mars 1954 à hauteur de 131000 francs (130 936 francs) pour des dégâts occasionnés à des bâtiments agricoles, étant précisé que ceux-ci étaient liés à l'ancienne ferme du Chalet. À noter que la localisation sur le territoire de cette propriété est déjà source d'interrogation. L'origine du préjudice est spécifiée : il s'agit bien du bombardement du 17 août 1944. Le rapport de la commission consultative précise toutefois que ces bâtiments ne renfermaient ni matériel ni cheptel.

Pour un précédent bombardement intervenu en juin de la même année, et pour l'autre partie de la ferme du Chalet toujours en activité, un dénommé Rosselle Omer, résidant à Proverville, reçoit, en octobre 1960, une indemnité de 355 NF (354,96 Nouveaux Francs) pour une perte de cheptel vif estimé à moins de $1200 \mathrm{NF}$ (1 183,20 NF). Si l'on rapporte ce 
montant à la valeur moyenne d'un cheval (430 NF : valeur février 1944), il ne peut s'agir que de deux ou trois chevaux d'exploitation.

$\mathrm{Au}$ lendemain de la guerre, aucun fait pouvant concerner une cinquantaine de chevaux n'a donc été rapporté, ni à la commission des dommages de guerre, ni au maire de Barsur-Aube. Si le cheptel des Varennes est bien contemporain de cette période, il ne peut s'agir que de chevaux ayant appartenu à l'armée allemande.

\section{Un nécessaire retour à la discipline}

39 Étrangement confrontée à des difficultés d'interprétation de l'origine et du sort des chevaux des Varennes par le seul biais de la recherche historique, notre quête d'une vérité imposait un retour au gisement archéologique et à une pratique de la discipline à partir des seules pièces et informations recueillies sur le terrain de la fouille, comme nous aurions pu l'envisager d'emblée pour un gisement paléolithique ou néolithique.

Ont donc été confiés à l'archéozoologue Alessio Bandelli (Inrap) l'étude des ossements prélevés et à l'anthropologue Sandrine Thiol (Inrap) le soin d'une réflexion sur la taphonomie et les origines probables de la mort de ces équidés.

\section{Archéozoologie}

\section{La composition et la qualité de l'échantillon}

4130 équidés au minimum sont documentés dans les différents tronçons de la tranchée explorés dans le cadre de l'opération. Plus précisément, 18 sujets sont illustrés dans les parties de la structure fouillées manuellement, et les os de pas moins de 12 autres individus ont été collectés dans les segments fouillés à la pelle mécanique. L'étude concerne essentiellement les 18 ensembles dégagés à la main (fig. 8). 
Figure 8

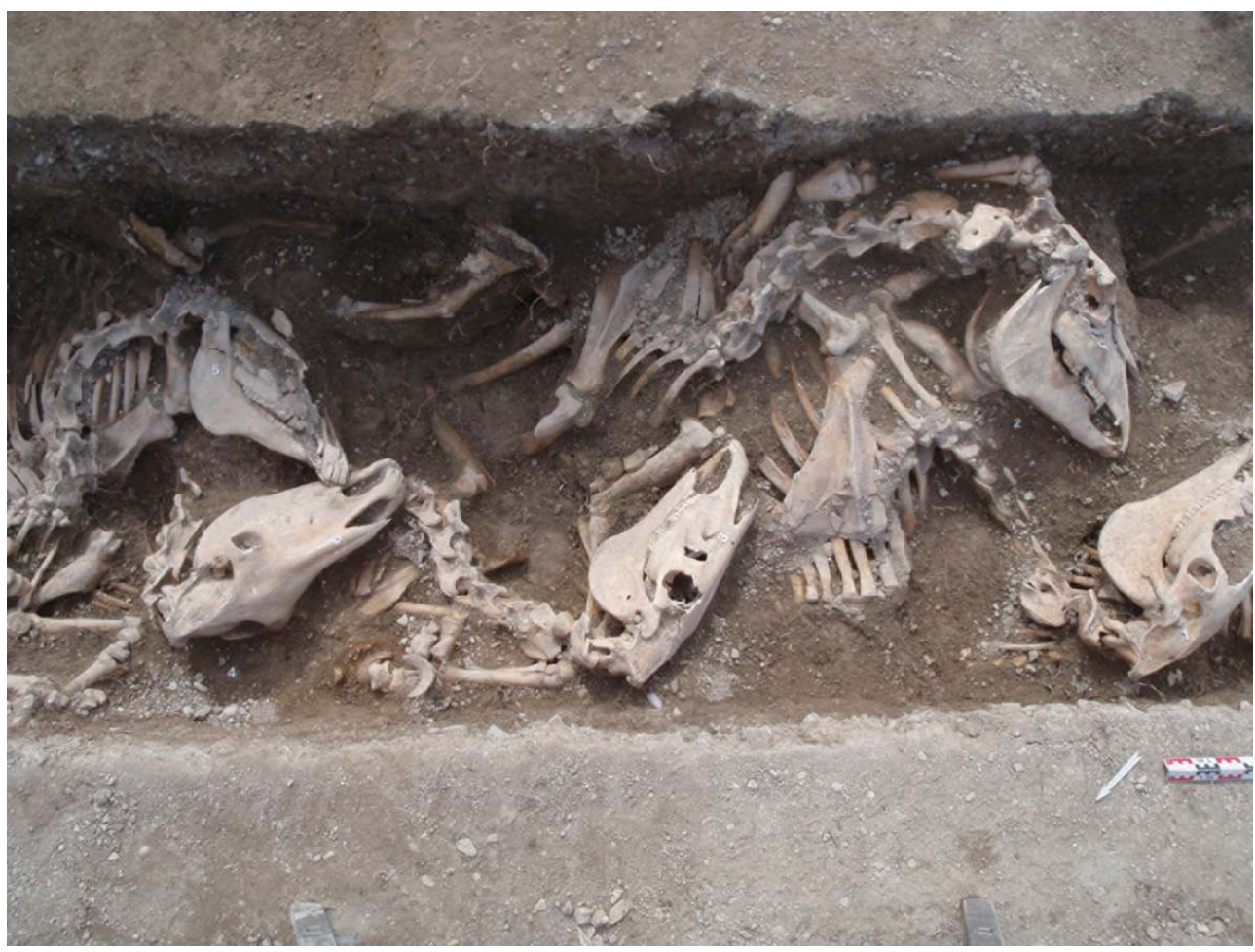

Mise en évidence des connexions anatomiques et des indices taphonomiques lors du dégagement manuel des carcasses.

Phot. Deborde, Gilles. (c) Inrap, 2014.

\section{La taxonomie, le sexe, l'âge et la stature}

L'examen des éléments anatomiques permet de confirmer la présence exclusive d'équidés dans la tranchée (cheval, âne, mulet ou bardot). L'analyse des dentitions des animaux a fourni des informations sur le sexe (canines) et sur l'âge (degré de développement des tables dentaires). Il ressort que, des 18 squelettes fouillés manuellement, 11 au moins correspondent à des mâles et 5 au moins à des femelles. Les animaux étaient, pour la plupart des adultes ( 1 individu d'au moins 16 ans, 10 individus entre 9 et 13 ans, 3 individus âgés entre 6 et 9 ans et 3 autres individus adultes qu'il n'a pas été possible d'évaluer précisément). Un sujet immature, âgé d'environ 3 ans, est également documenté.

Les principaux os des membres des individus présents dans la section 141 et quelques fémurs collectés dans les autres sections ont fait l'objet de mensurations qui permettent d'évoquer des sujets de grands gabarits comparables à ceux de chevaux de trait actuels («Draft », « Hannover » ou «boulonnais »). Toutefois, quelques rares os se caractérisent par des dimensions plus proches de celles de grands chevaux de selle de type arabe ${ }^{11}$ (fig. 9). 
Figure 9

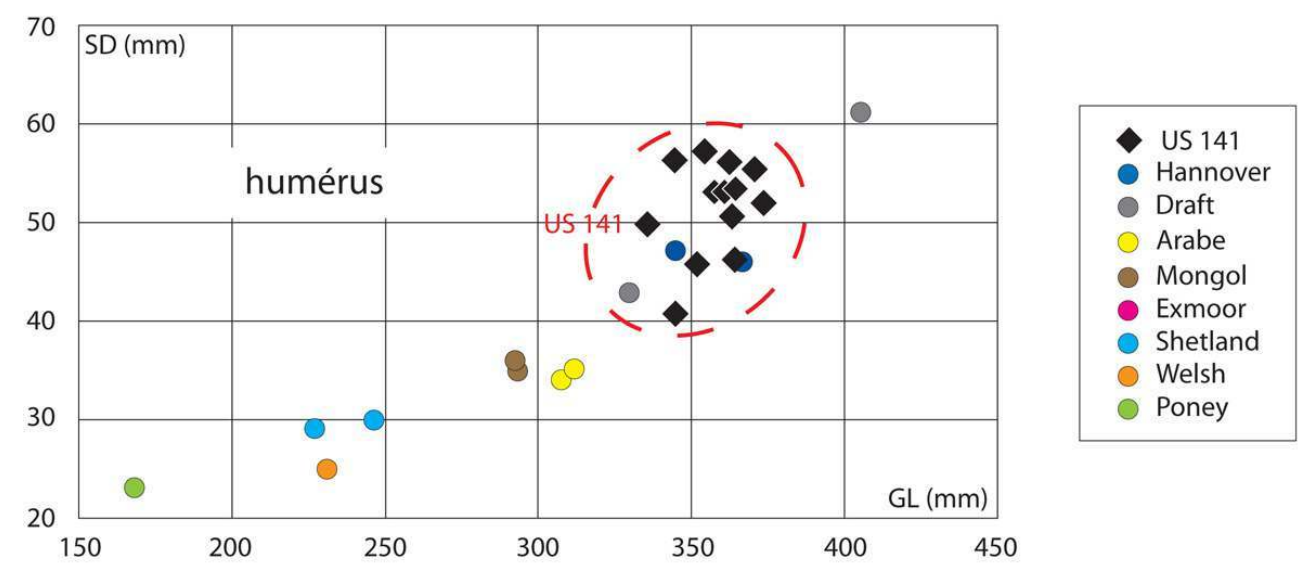

Projection sur le diagramme de référence de Vera Eisenmann des mesures prises sur les fémurs des chevaux des Varennes.

Conception Bandelli, Alessio, (c) Inrap, 2014.

\section{L'état sanitaire}

Plusieurs observations permettent d'évoquer un certain nombre de problèmes d'ordre sanitaire. Des affections des tables dentaires ont concerné deux individus présentant des irrégularités d'usure de certaines dents jugales. Des pathologies osseuses articulaires, susceptibles d'avoir été déclenchées ou aggravées par de fortes sollicitations mécaniques $\mathrm{du}$ squelette, ont touché d'autres sujets. Des ankyloses des vertèbres lombaires s'observent sur un individu et un autre présente une fusion pathologique des deux dernières vertèbres thoraciques ainsi qu'une exostose sur le condyle médial de son fémur gauche.

Les phalanges distales de quelques individus se signalent par la présence de proliférations osseuses sur les processus palmaires, traduisant différents degrés d'ossification des cartilages. Aujourd'hui, cette affection concerne principalement les phalanges des chevaux de trait. Il convient de remarquer ici qu'une des phalanges concernées portait un fer à plaque (voir fig. 5). Un emplâtre d'argile épousant l'empreinte de la phalange a également été retrouvé à proximité. Il est probable que ce type de fer avait une fonction thérapeutique.

Enfin, des exostoses sur les diaphyses d'un métacarpe d'un individu et d'un tibia d'un autre, résultant probablement d'inflammations du périoste, sont également recensées.

Sur le crâne du cheval mâle $\mathrm{n}^{\circ} 1$, une petite dent pointe au milieu du diastème de la mâchoire supérieure de l'animal (fig. 10). Il s'agit d'une dent « vestigiale » ou « dent-deloup ", apparaissant sur une minorité de sujets, qui est arrachée ou limée pour pouvoir passer le mors. Sa présence peut intriguer sur des animaux de trait ou de monte. Lorsqu'elle a été limée, cette dent peut repousser lors d'une phase de repos prolongé de l'animal. Elle peut également caractériser un animal spécialement réservé à la reproduction. 
Figure 10

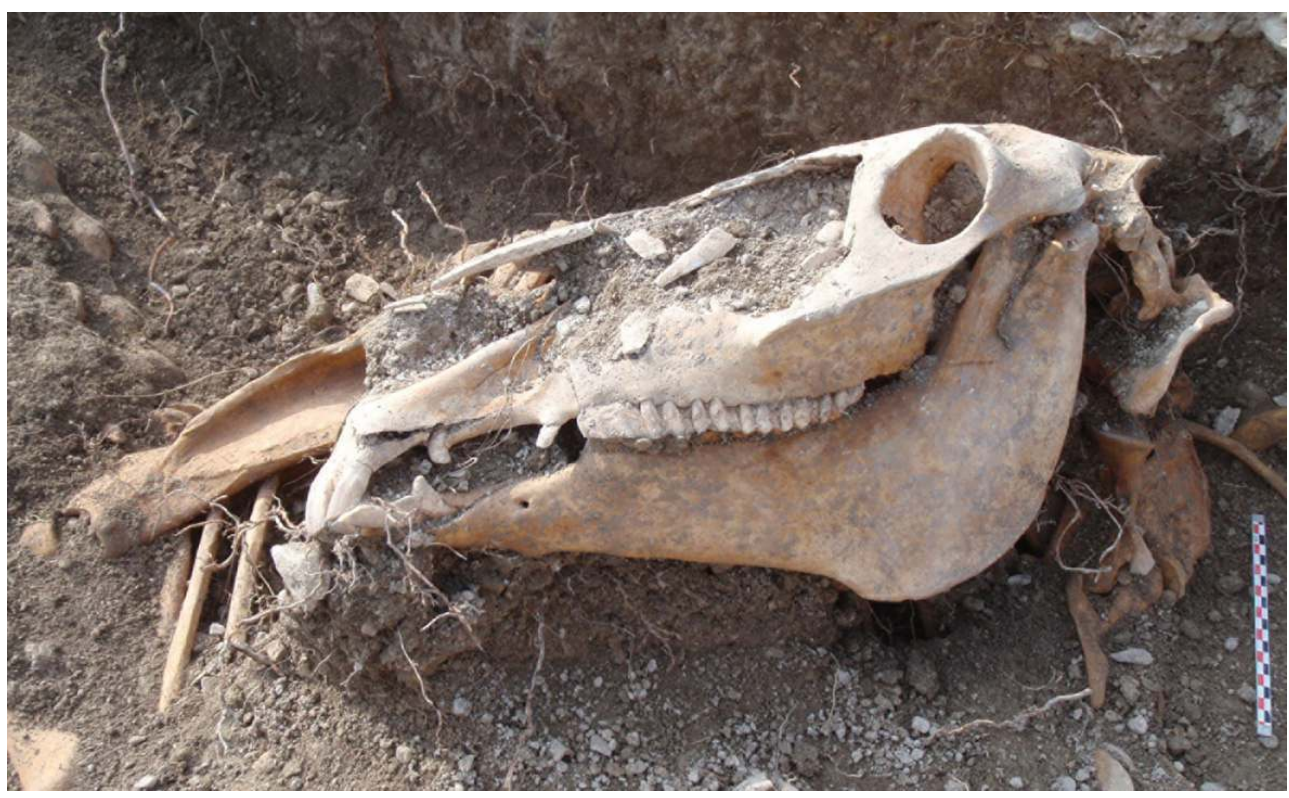

« Dent de loup », canine vestigiale présente dans la dentition de l'individu mâle n 1 .

Phot. Deborde, Gilles. (c) Inrap, 2014.

\section{La manipulation des cadavres} désarticulation partielle des bas des pattes de quatre individus. C'est, très vraisemblablement, lors de la mise en terre que ces animaux ont fait l'objet de manipulations. Pour un individu, ces traces se présentent sous la forme d'incisions provoquées par un couteau et portées sur l'extrémité distale du radius droit. Pour un autre, l'action de désarticulation est documentée sur l'extrémité distale du tibia gauche et $\mathrm{du}$ talus attenant, par plusieurs incisions profondes provoquées par un outil tranchant lourd. Chez un troisième individu, c'est son talus droit qui porte les traces d'une découpe du tarse, pratiquée au couteau. Chez un quatrième enfin, les quatre bas de pattes sont concernés par une découpe.

Il est probable que ces manipulations aient été effectuées pour faciliter l'introduction ou améliorer l'agencement dans la tranchée des corps des chevaux. Elles auraient été nécessaires à cause de l'état de rigidité cadavérique des animaux, soit au plus tard quelques jours après la mort des bêtes.

Enfin, signalons que l'individu $\mathrm{n}^{\circ} 1$ présente une fracture non consolidée du radius gauche. Celle-ci a vraisemblablement été provoquée par le choc de l'os contre le bord du fossé, ce qui évoque l'introduction en force du corps de l'animal dans la structure (fig. 11) 
Figure 11

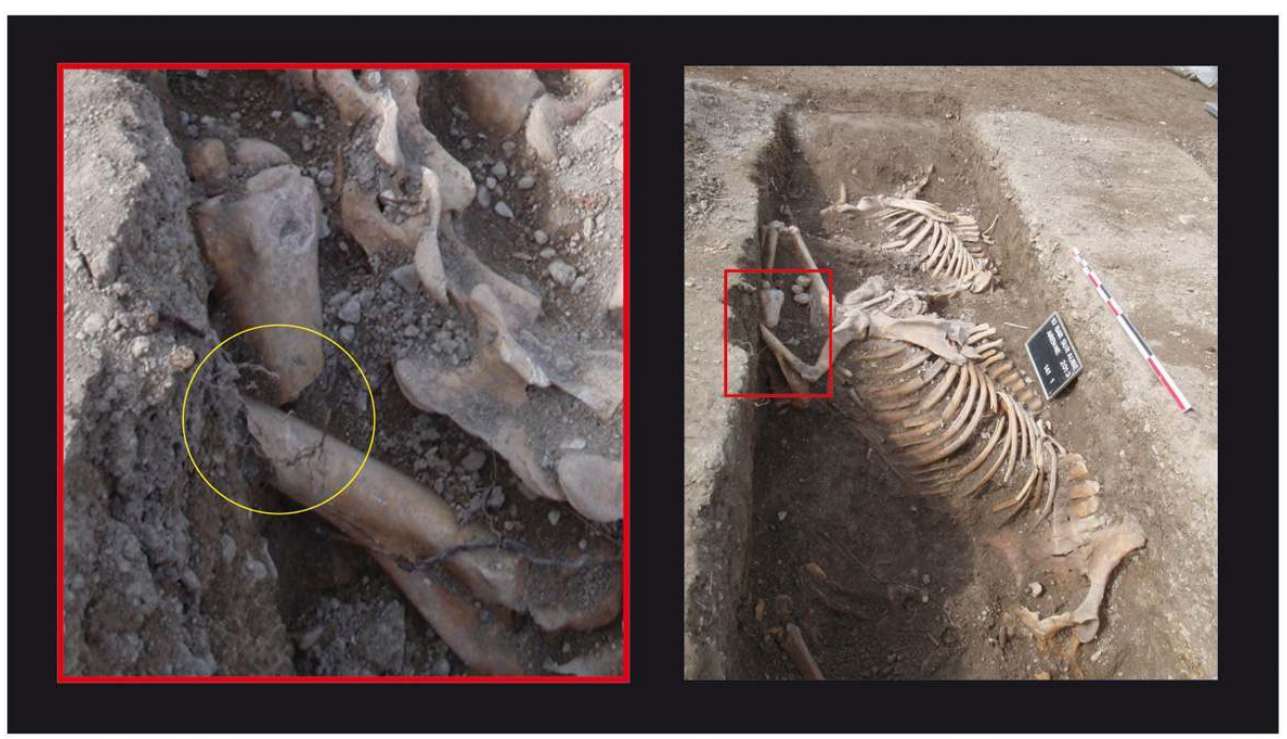

Vue de détail du radius gauche de l'individu $n^{\circ} 1$, vraisemblablement fracturé au moment de la dépose de l'animal dans la tranchée.

Phot. Deborde, Gilles. (c) Inrap, 2014.

\section{Taphonomie et médecine légale}

\section{Les indices de mort violente}

Si un même événement catastrophique peut être à l'origine de la mort simultanée d'au moins 18 de ces animaux, des indices archéozoologiques sont susceptibles de contribuer à la reconstitution des circonstances de ces décès. Il s'agit de traces de mise à mort, sous la forme de perforations observées sur trois crânes. Pour l'individu $\mathrm{n}^{\circ} 1$ cette perforation se situe sur l'os pariétal gauche. Le contour de la perforation s'inscrit dans un losange et pourrait avoir été façonné par un objet pointu. Les traces repérées sur les crânes des individus $n^{\circ} 7$ et $n^{\circ} 9$ se trouvent au même endroit que celle observée sur l'individu $n^{\circ} 1$ et peuvent correspondre à des impacts de balles de 6,5 et $8,5 \mathrm{~mm}$ ayant pénétré l'os suivant une trajectoire inclinée (fig. 12). Le crâne de l'individu ${ }^{\circ} 9$ a été atteint par un projectile qui a perforé l'os frontal droit pour ensuite traverser la boîte crânienne et ressortir à travers l'os basi-sphénoïde droit. 


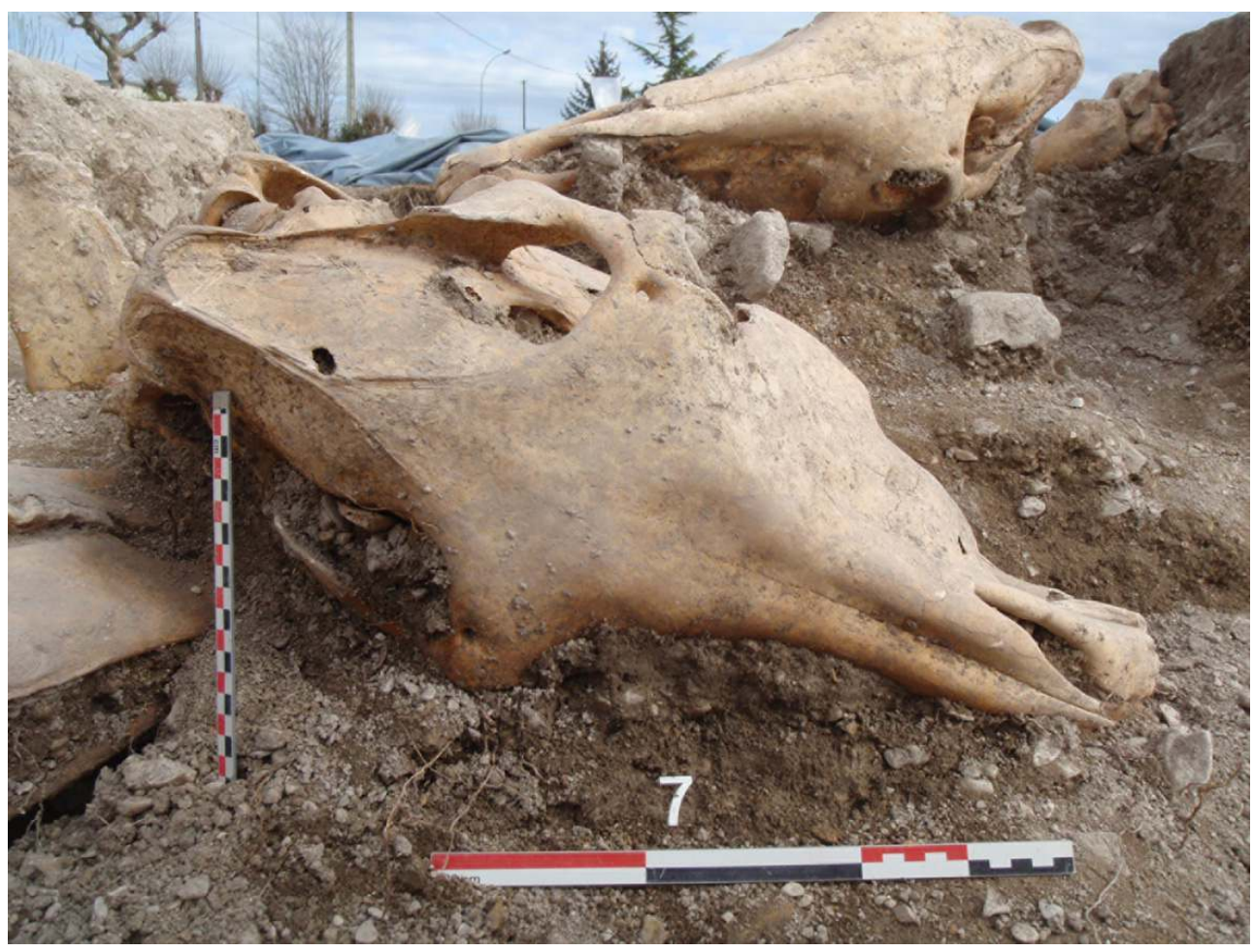

Trace de mise à mort sur la boîte crânienne de l'individu $n^{\circ} 7$

Phot. Deborde, Gilles. (c) Inrap, 2014.

\section{Les circonstances de la mort}

La disposition des cadavres dans la section de tranchée 141 et les indices de manipulation post-mortem des animaux conduisent à retenir la forte probabilité d'un état de rigidité cadavérique et donc d'un enfouissement au plus tard quelques jours après leur mort.

L'état sanitaire du cheptel renvoie aux pathologies osseuses articulaires décrites sur les fiches de signalement des chevaux réquisitionnés, pathologies qui caractérisent généralement les animaux soumis à un travail prolongé. Aucun des 41 chevaux ne présente toutefois de traces de fractures qui auraient été invalidantes ou directement liées aux causes de la mort. Ces équidés n'ont pas été tués dans un bombardement qui aurait laissé de telles séquelles, disloqué les squelettes et occasionné la présence d'éclats à l'intérieur des corps. Ils n'ont pas été non plus été sacrifiés car seuls quelques individus présentent des indices de mise à mort par balles et/ou autres objets perforants et aucun des corps n'a retenu de projectile. Les impacts se situant préférentiellement sur le haut du crâne, on peut supposer que l'animal, de grand gabarit, était à terre lorsqu'il reçut un « coup de grâce » destiné à mettre fin à une agonie.

Un phénomène particulier peut engendrer un état équivalent à la rigidité cadavérique et, comme elle, maintenir une perte d'élasticité de la peau et des muscles jusqu'au déclenchement du processus de décomposition. Il s'agit de la chaleur. Face à l'hypothèse d'un incendie, nous sommes encore confrontés à une absence d'indices clairs, notamment de traces de carbonisation superficielle causées par les flammes au niveau des régions anatomiques faiblement recouvertes de chair (crâne et vertèbres). Mais il est possible 
d'envisager les conséquences d'une asphyxie par des fumées qui auraient envahi les écuries où étaient retenus ces chevaux. Ceux retrouvés agonisant à l'issue du sinistre auraient alors été abattus par un coup mortel porté sur le crâne.

\section{L'origine des chevaux de Bar-sur-Aube}

La tranchée de défense passive ouverte sur le site des Varennes au cours de la Seconde Guerre mondiale était bien trop importante pour assurer la seule protection des ouvriers de l'usine d'estampage située de l'autre côté de la rue. Elle devait être destinée à d'autres fins, certainement militaires. Elle a servi de sépulture à plus de quarante chevaux en âge de travailler ( 3 ans et plus) après qu'elle ait perdu de son utilité, c'est-à-dire après le départ des troupes allemandes auxquelles elle était vraisemblablement destinée. L'état sanitaire des bêtes, probablement toutes enfouies sur un laps de temps très court, correspond à un cheptel sain, sélectionné par rapport à une population équine alors fatiguée et meurtrie par une forte sollicitation au cours de la période de l'Occupation. Il s'agit de bêtes réquisitionnées retenues et soignées à proximité du site des Varennes, vraisemblablement à la ferme du Chalet. La présence d'étalons susceptibles d'avoir été employés comme reproducteurs accrédite l'hypothèse d'un centre de soin mais également de sélection.

Le 27 août 1944, alors que Troyes vient d'être libéré, les troupes allemandes pillent la ville de Bar-sur-Aube et retiennent en otage pendant 3 jours une dizaine de civils qu'ils libèreront avant de battre en retraite le 30 août. On peut comprendre le trouble subi alors par la population baralbine pour admettre que la mort de quarante chevaux, hors la ville, ait pu sembler dérisoire ou passer inaperçue. Les circonstances de cette hécatombe (incendie ou empoisonnement) restent imprécises mais la présence d'une garnison allemande entre décembre 1943 et août 1944 sur le site de la ferme du Chalet, proche des Varennes, est plus que probable, malgré l'absence totale de témoignage sur le sujet. Les troupes d'occupation devaient y être employées à la sélection et à l'entretien d'un cheptel équin au profit de sa seule armée.

\section{NOTES}

1. - TRINQUET, Jean-Claude et al. Les usines Morvan. Voir le site $: \mathrm{http} / / /$ www.ventsdumorvan.org.

2. - HAMMAN, Philippe. La belle histoire des assiettes: Sarreguemines, 1836-1918. Metz: Éd. Serpenoise, 2007, p. 94-98.

3. - Information : Duchene, Bruno / Inrap GEN.

4. - Voir le site : http://www.inrap.fr.

5. - Arch. dép. Aube, 3 P 5181.

6. - Ibid., 3 P 894.

7. - Ibid., 310 W 64.

8. - Ibid,, 310 W 135. 
9. - ARCELIN, Gérard. Chronique des années noires dans l'arrondissement de Bar-sur-Aube, 1939-1945. Bar-sur-Aube : Némont éditeur-imprimeur, 1989, p. 432.

10. - Arch. dép. Aube, 1021 W 54.

11. - Voir le site : http://www.vera-eisenmann.com.

\section{RÉSUMÉS}

La mise au jour en 2013, à Bar-sur-Aube (Aube), par une équipe de l'Inrap, d'une tranchée remplie de plus d'une quarantaine de carcasses d'équidés est à l'origine d'une enquête archéologique et historique qui a conduit les archéologues à explorer de nombreuses pistes. Confrontés à l'absence de témoignages vivants et documentaires directs, ils ont exploité les ressources des deux disciplines pour enfin pouvoir associer ces carcasses à une ultime campagne de réquisition entreprise par les troupes d'occupation dans le département en août 1944. Une grande partie de ces chevaux devait être parquée dans un bâtiment de ferme situé à proximité et occupé par l'armée allemande. Un violent incendie peut être à l'origine de cette hécatombe.

Horses under the Occupation, the mass grave at the Varennes archaeological at Bar-sur-Aube (Aube). The discovery in 2013, at Bar-sur-Aube of a trench filled with more than forty carcasses of horses, led the team of archaeologists who made this discovery to explore a number of archaeological and historical lines of enquiry. For lack of direct witnesses or any documentary record, they used the resources of the two disciplines in order to understand that these horses had been requisitioned by the German occupying forces in August 1944. Most of these horses were stabled in a farm building nearby, occupied by the Germans. A violent fire was possibly the cause of the horses' death.

\section{INDEX}

Mots-clés : Bar-sur-Aube, tranchée, chevaux, fer à cheval, Occupation, réquisition, Seconde Guerre mondiale, archéozoologie

Keywords : trench, horse, horseshoe, Second World War, archeo-zoology

\section{AUTEUR}

\section{GILLES DEBORDE}

Institut national de recherches archéologiques préventives / responsable d'opérations et d'études / avec la collaboration d'Alessio Bandelli (Inrap) et Sandrine Thiol (Inrap)

gilles.deborde@inrap.fr 